\title{
On overgroups of regular abelian $p$-groups
}

\author{
Edward Dobson \\ Department of Mathematics and Statistics \\ Mississippi State University \\ PO Drawer MA Mississippi State, MS 39762, USA
}

Received 3 July 2008, accepted 28 January 2009, published online 26 March 2009

\begin{abstract}
Let $G$ be a transitive group of odd prime-power degree whose Sylow $p$-subgroup $P$ is abelian of rank $t$. We show that if $p>2^{t-1}$, then $G$ has a normal subgroup that is a direct product of $t$ permutation groups of smaller degree that are either cyclic or doublytransitive simple groups. As a consequence, we determine the full automorphism group of a Cayley digraph of an abelian group with rank two such that the Sylow $p$-subgroup of the full automorphism group is abelian.
\end{abstract}

Keywords: Cayley graph, abelian group, regular group, p-group

Math. Subj. Class.: 20B10, 05C25

\section{Introduction}

In this paper, we study transitive groups of odd prime-power degree whose Sylow $p$ subgroup is abelian with rank $t$. In the case of $t=2$, we explicitly determine them (Corollary 6.5). Our main motivation is to determine the full automorphism group $\operatorname{Aut}(\Gamma)$ of Cayley digraphs $\Gamma$ of abelian groups $G$ such that the full Sylow $p$-subgroup of the automorphism group is abelian (and hence $G_{L}$ and also as small as possible). This is an important case in trying to determine the full automorphism group of arbitrary Cayley digraphs of $G$, as a "large" Sylow $p$-subgroup usually has quite a bit of structure (and so in many cases it's overgroups can be determined). This occurs because if $\operatorname{Aut}(\Gamma)$ has a large Sylow $p$-subgroup, then usually $\Gamma$ has many edges that occur in a structured way for example $\Gamma$ can be written as a wreath product or perhaps a "semiwreath" product. As a concrete example of this type of behavior, it has been shown that a Cayley digraph $\Gamma$ of an abelian group $G$ of order $p^{2}$ has automorphism group with Sylow $p$-subgroup either isomorphic to $G$ or to $\mathbb{Z}_{p}\left\{\mathbb{Z}_{p}[1,10]\right.$. In the latter case, a classical result of Sabidussi [18]

E-mail address: dobson@math.msstate.edu (Edward Dobson) 
gives $\operatorname{Aut}(\Gamma)$, so determining $\operatorname{Aut}(\Gamma)$ reduces to the case where the Sylow $p$-subgroup of $\operatorname{Aut}(\Gamma)$ is $G$.

Historically, there has been some work on explicitly determining the transitive groups of prime power degree given that its Sylow $p$-subgroup is abelian. Chronologically, the first such result was obtained by Burnside [2] in 1901, who showed that a transitive group of prime degree $p$ is either doubly-transitive or contains a normal Sylow $p$-subgroup (which is necessarily cyclic as the degree is prime). The author generalized this result [7] and showed that a transitive group of odd prime-power degree $p^{k}$ such that every minimal transitive subgroup is cyclic is either doubly-transitive or has a normal Sylow $p$-subgroup. Building on work of Wielandt [20], Jones [14] showed in 1979 that a transitive group of degree $p^{2}$ with elementary abelian Sylow $p$-subgroup contains a normal subgroup that is permutation isomorphic to a direct product of two groups, with the canonical action, each of which is either cyclic or doubly-transitive and simple (and so this result of Jones can be thought of as a generalization of Burnside's result mentioned above). In this paper, we will first generalize Jones' result to an odd prime-power $p^{k}, k \geq 2$, provided that the transitive permutation group contains a Sylow $p$-subgroup that is abelian with rank $t$ (Theorem 6.4) and $p>2^{t-1}$. Note that as $p$ is odd, this condition is always satisfied if $t=2$, and so for abelian groups with rank two, we generalize Jones' result with no restrictions on $p$ other than $p$ is odd. We will then explicitly determine all transitive permutation groups of odd prime-power $p^{k}$ whose Sylow $p$-subgroup is abelian of rank two (Corollary 6.5), and finally, we determine all possible automorphism groups of Cayley digraphs of abelian groups of rank two whose Sylow $p$-subgroups are abelian (Corollary 7.3).

As the proof of the main result is somewhat long, it will be useful to provide a general outline of the proof. Let $G$ be a transitive group of odd prime-power degree $p^{k}, p$ an odd prime, with an abelian Sylow $p$-subgroup $P$. If $G$ is primitive, then one can use the O'NanScott Theorem together with the Classification of the Finite Simple Groups to analyze $G$. This has already been done - $\mathrm{Li}$ [16] has determined all primitive groups that contain a regular abelian subgroup. We are then left with the case where $G$ is imprimitive. We choose $\mathcal{B}$ to be a complete block system of $G$ such that $G$ admits no nontrivial complete block system whose blocks are strictly contained within blocks of $\mathcal{B}$. Then the set-wise stabilizer of a block of $\mathcal{B}$ in $G$, denoted $\operatorname{Stab}_{G}(B)$, is primitive in its action on $B$ [5, Exercise 1.5.10]. Thus these groups are given by [16], and fall into three classes: almost simple groups, affine groups, and primitive groups constructed by the product action. We will also need to use the set-wise stabilizer of every block of $\mathcal{B}$, denoted $\operatorname{fix}_{G}(\mathcal{B})$, in our analysis. Combined with the above possibilities for the action of $\operatorname{Stab}_{G}(\mathcal{B})$ on $B \in \mathcal{B}$, we have the following possibilities. Both $\operatorname{Stab}_{G}(B)$ and $\left.\operatorname{fix}_{G}(\mathcal{B})\right|_{B}$ are almost simple, affine, or primitive groups constructed via the product action, as well as the case where $\operatorname{Stab}_{G}(B)$ is primitive on $B$ but $\left.\operatorname{fix}_{G}(\mathcal{B})\right|_{B}$ is imprimitive. In the former three cases, we show that action of $\operatorname{fix}_{G}(\mathcal{B})$ on $B$ is equivalent to the action of $\operatorname{fix}_{G}(\mathcal{B})$ on $B^{\prime}$ (these actions are considered in Section 3), $B, B^{\prime} \in \mathcal{B}$, and then use relatively standard techniques to show that $G$ is contained in a canonical direct product provided that $\operatorname{fix}_{G}(\mathcal{B})$ is not semiregular. The case where $\operatorname{fix}_{G}(\mathcal{B})$ is semiregular is considered in Section 4, while the case $\operatorname{Stab}_{G}(B)$ is primitive on $B$ but $\left.\operatorname{fix}_{G}(\mathcal{B})\right|_{B}$ is imprimitive is considered in Lemma 6.2. The main results are then proven in Section 6.

Definition 1.1. Let $G$ be a finite abelian group, so that $G \cong \mathbb{Z}_{p_{1}^{a_{1}}} \times \mathbb{Z}_{p_{2}^{a_{2}}} \times \cdots \times \mathbb{Z}_{p_{r}^{a_{r}}}$ in accordance with the Fundamental Theorem of Finitely Generated Abelian Groups. We define the rank of $G$ to be $r$. 
The following result is [7, Theorem 33].

Theorem 1.2. Let $p \geq 3$ be prime, and $G \leq S_{p^{m}}, m \geq 1$, be transitive such that every minimal transitive subgroup of $G$ is cyclic. Then either $G$ contains a transitive normal Sylow p-subgroup, or $G$ is doubly-transitive and

1. $G=A_{p^{m}}$ or $S_{p^{m}}$, and $m=1$,

2. $\operatorname{PSL}(n, k) \leq G \leq \operatorname{P\Gamma L}(n, k)$, for some prime power $k$ and $n \geq 2$ with $p^{m}=$ $\left(k^{n}-1\right) /(k-1)$,

3. $G=\operatorname{PSL}(2,11)$ or $M_{11}$ and $p^{m}=11$,

4. $G=M_{23}$ and $p^{m}=23$.

Definition 1.3. For a positive integer $n$, define $N(n)=\left\{x \rightarrow a x+b: a \in \mathbb{Z}_{n}^{*}, b \in \mathbb{Z}_{n}\right\}$. Thus $N(n)$ is the normalizer of the left regular representation $\left(\mathbb{Z}_{n}\right)_{L}$ of $\mathbb{Z}_{n}$ in $S_{n}$. In general, for a group $G$, we define $G_{L}=\{h \rightarrow g h: h, g \in G\}$. We remark that for $p$ a prime, $N(p)$ is usually denoted $\operatorname{AGL}(1, p)$.

Definition 1.4. Let $G \leq S_{n}$ be imprimitive with complete block system $\mathcal{B}$. We say that $\mathcal{B}$ is normal if $\mathcal{B}$ is formed by the orbits of a normal subgroup of $G$. Also, any $g \in G$ permutes the blocks of $\mathcal{B}$, and we denote this induced permutation by $g / \mathcal{B}$. We set $G / \mathcal{B}=$ $\{g / \mathcal{B}: g \in G\}$, and $\operatorname{fix}_{G}(\mathcal{B})=\{g \in G: g(B)=B$ for all $B \in \mathcal{B}\}$. If $\mathcal{C}$ is another complete block system of $G$, we write $\mathcal{C} \preceq \mathcal{B}$ if every block of $\mathcal{B}$ is a union of blocks of $\mathcal{C}$. If $\mathcal{C} \preceq \mathcal{B}$, then $G / \mathcal{C}$ admits a complete block system, denoted $\mathcal{B} / \mathcal{C}$, induced by $\mathcal{B}$. That is each block of $\mathcal{B} / \mathcal{C}$, say $B / \mathcal{C}$, is the set of those blocks of $\mathcal{C}$ whose union is $B$. For a point $i$, we denote the point-wise stabilizer of $i$ in $G$ by $\operatorname{Stab}_{G}(i)$, and for a subset $B$ of $\mathbb{Z}_{n}$ (we assume that $G$ acts on $\mathbb{Z}_{n}$ ), we denote the set-wise stabilizer of $B$ in $G$ by $\operatorname{Stab}_{G}(B)$.

\section{Some General Results}

The following general results on permutation groups will be useful.

Definition 2.1. For a permutation group $H \leq S_{\Omega}$, we define the support of $H$, denoted $\operatorname{supp}(H)$, to be the set of all $x \in \Omega$ such that there exists $h \in H$ such that $h(x) \neq x$.

Lemma 2.2. Let $G \leq S_{n}$ be transitive such that $G$ admits a complete block system $\mathcal{B}$ consisting of $m$ blocks of size $k$. If $T=\left.\operatorname{soc}\left(\operatorname{fix}_{G}(\mathcal{B})\right)\right|_{B}$ is a transitive nonabelian simple group, then $\mathcal{C}=\left\{\operatorname{supp}(M): M\right.$ is a minimal normal subgroup of $\left.\operatorname{fix}_{G}(\mathcal{B})\right\}$ is a complete block system of $G, \mathcal{B} \preceq \mathcal{C}$, and $\operatorname{soc}\left(\operatorname{fix}_{G}(\mathcal{B})\right)$ is a direct product of simple groups isomorphic to $T$.

Proof. As $\operatorname{soc}\left(\left.\operatorname{fix}_{G}(\mathcal{B})\right|_{B}\right)$ is a transitive nonabelian simple group, a minimal normal subgroup $M$ of $\operatorname{fix}_{G}(\mathcal{B})$ is nonabelian. In fact, if $\left.M\right|_{B} \neq 1$, then $\left.M\right|_{B}$ contains the socle of $\left.\operatorname{fix}_{G}(\mathcal{B})\right|_{B}$ for $B \in \mathcal{B}$, and so if $\left.M\right|_{B} \neq 1$, then $\left.M\right|_{B}$ is a transitive nonabelian simple group. Let $N$ be another minimal normal subgroup of $\operatorname{fix}_{G}(\mathcal{B})$. Note then that $\operatorname{supp}(M)$ and $\operatorname{supp}(N)$ are unions of blocks of $\mathcal{B}$. If $B_{1}, B_{2} \in \mathcal{B}$ with $B_{1} \subseteq \operatorname{supp}(M)$ and $B_{2} \subseteq \operatorname{supp}(N)$, then as minimal normal subgroups centralize each other, we have that $B_{1} \cap B_{2}=\emptyset$. Define a relation $\approx$ on $\mathbb{Z}_{n}$ by $i \approx j$ if and only if $i, j \in C \in \mathcal{C}$. Note that as $G$ is transitive, $\approx$ is reflexive. That $\approx$ is symmetric and transitive are trivial, and so $\approx$ is an equivalence relation. Let $g \in G$, and $i \approx j$. Then $g M g^{-1}$ is a minimal normal subgroup 
of $\operatorname{fix}_{G}(\mathcal{B})$, and $g(i)$ and $g(j)$ are contained in $\operatorname{supp}\left(g M g^{-1}\right)$. This last statement follows as, if $i, j \in \operatorname{supp}(M)$, there exists $h_{i}, h_{j} \in M$ such that $h_{i}(i) \neq i$ and $h_{j}(j) \neq j$. Then $g h_{i} g^{-1}(g(i))=g\left(h_{i}(i)\right) \neq g(i)$ as $h_{i}(i) \neq i$. Similarly, $g h_{j} g^{-1}(g(j)) \neq g(j)$. Thus $\approx$ is a $G$-congruence, and by [5, Exercise 1.5.4], $\mathcal{C}$ is a complete block system of $G$. As $\mathcal{C}$ is a complete block system of $G$, we have that any two minimal normal subgroups of $\operatorname{fix}_{G}(\mathcal{B})$ are conjugate and so isomorphic, and it is easy to see that a minimal normal subgroup must be isomorphic to $\operatorname{soc}\left(\left.\operatorname{fix}_{G}(\mathcal{B})\right|_{B}\right)$.

Lemma 2.3. Let $G \leq S_{m p^{k}}$, $p$ a prime, $k \geq 1$, be such that $G$ admits a complete block system $\mathcal{B}$ consisting of $m$ blocks of size $p^{k}$ such that $\operatorname{fix}_{G}(\mathcal{B})$ contains a semiregular element $\rho$ of order $p^{k}$. Additionally, assume that $\left.\operatorname{fix}_{G}(\mathcal{B})\right|_{B}$ is doubly-transitive with nonabelian socle and has a cyclic Sylow p-subgroup $\left\langle\left.\rho\right|_{B}\right\rangle, B \in \mathcal{B}$. Then there exists a complete block system $\mathcal{B} \preceq \mathcal{C}$ such that a Sylow p-subgroup of $\operatorname{fix}_{G}(\mathcal{B})$ is $\left\langle\left.\rho\right|_{C}: C \in \mathcal{C}\right\rangle$.

Proof. By Lemma 2.2, we have that $\mathcal{C}=\{\operatorname{supp}(M): M$ is a minimal normal subgroup of fix $\left.\operatorname{fin}_{G}(\mathcal{B})\right\}$ forms a complete block system of $G$, consisting of, say, $n$ blocks of size $r p^{k}$, and $\mathcal{B} \preceq \mathcal{C}$. Let $M_{1}, \ldots, M_{n}$ be distinct minimal normal subgroups of $\operatorname{fix}_{G}(\mathcal{B})$, so that $\mathcal{C}=\left\{\operatorname{supp}\left(M_{i}\right): 1 \leq i \leq n\right\}$. Let $P$ be a Sylow $p$-subgroup of $\operatorname{fix}_{G}(\mathcal{B})$ that contains $\langle\rho\rangle$. As each $M_{i}$ is transitive on some $B_{i} \in \mathcal{B}$, we have that $p^{k}$ divides $\left|M_{i}\right|$ for $1 \leq i \leq n$. Also, if $B_{i} \subseteq \operatorname{supp}\left(M_{i}\right), B_{i} \in \mathcal{B}$, then $M_{i}$ must act faithfully on $B_{i}$ or $M_{i}$ is not a minimal normal subgroup. Thus a Sylow $p$-subgroup $P_{i}$ of $M_{i}$ has order $\left|P_{i}\right|_{B_{i}} \mid$. As a Sylow $p$-subgroup $\left.\operatorname{fix}_{G}(\mathcal{B})\right|_{B}$ is cyclic and regular, $\left.P_{i}\right|_{B_{i}}$ is cyclic and regular, and so a Sylow $p$-subgroup of $\Pi_{i=1}^{n} M_{i}$ has order $p^{n k}$. We assume without loss of generality that each $P_{i} \leq P$.

Now, let $\rho^{\prime} \in P$, and for each $M_{i}, 1 \leq i \leq n$, choose $B_{i} \in \mathcal{B}$ such that $B_{i} \subseteq$ $\operatorname{supp}\left(M_{i}\right)$. As $\left.\rho^{\prime}\right|_{B_{i}}$ is contained in a Sylow $p$-subgroup of $\left.\operatorname{fix}_{G}(\mathcal{B})\right|_{B_{i}}$, and all Sylow $p$ subgroups of $\left.\operatorname{fix}_{G}(\mathcal{B})\right|_{B_{i}}$ are conjugate and so contained in $\left.M_{i}\right|_{B_{i}}$, we have that $\left.\rho^{\prime}\right|_{B_{i}} \in$ $M_{i}$. Also, as a Sylow $p$-subgroup of $\left.\operatorname{fix}_{G}(\mathcal{B})\right|_{B}$ is cyclic, $\left.\rho\right|_{B_{i}}$ is a Sylow $p$-subgroup of $\left.M_{i}\right|_{B_{i}}, \rho^{\prime} \in P$, and a transitive abelian group is self-centralizing, we have that $\left.\rho^{\prime}\right|_{B_{i}} \in$ $\left.\langle\rho\rangle\right|_{B_{i}}$. We conclude that there exists $\delta_{i} \in M_{i}$ such that $\left.\delta_{i} \rho^{\prime}\right|_{B_{i}}=1$. As $\left.\left.\delta_{i}\right|_{B_{i}} \in\langle\rho\rangle\right|_{B_{i}}$, we must have that $\delta_{i} \in\left\langle\left.\rho\right|_{\operatorname{supp}\left(M_{i}\right)}\right\rangle$ as otherwise $\left.\left\langle\delta_{i}, \rho\right\rangle\right|_{\operatorname{supp}\left(M_{i}\right)}$ does not act faithfully on $\operatorname{supp}\left(M_{i}\right)$, and so $M_{i}$ is not a minimal normal subgroup. Let $\delta=\Pi_{i=1}^{n} \delta_{i}$. Then $\left.\delta \rho^{\prime}\right|_{B_{i}}=1$ for every $1 \leq i \leq n$. Note that if $\delta \rho^{\prime}=1$, then the result follows, as then $P=\left\langle\delta_{i}: 1 \leq i \leq n\right\rangle$ and $\delta_{i} \in\left\langle\left.\rho\right|_{\operatorname{supp}\left(M_{i}\right)}\right\rangle$. If $\delta \rho^{\prime} \neq 1$, then $\left\langle\delta \rho^{\prime}\right\rangle^{\text {fix }}{ }^{(\mathcal{B})}$ is a normal subgroup of $\operatorname{fix}_{G}(\mathcal{B})$, and so contains a minimal normal subgroup $M$. Furthermore, there exists $B \in \mathcal{B}$ such that $\left.M\right|_{B} \neq 1$. As $\mathcal{C}$ is a complete block system of $G$, there exists $1 \leq i \leq n$ such that $\left.M_{i}\right|_{B}$ is transitive. Also, $M$ and $M_{i}$ centralize each other as they are minimal normal subgroups of $\operatorname{fix}_{G}(\mathcal{B})$. However, $\left.M_{i}\right|_{B}$ and $\left.M\right|_{B}$ are transitive nonabelian groups with simple socle, and as they both contain a transitive cyclic subgroup, they are not regular, contradicting [5, Theorem 4.1A (ii)]. The result then follows.

\section{The number of inequivalent actions of certain groups}

In order to prove our main results, we shall need to know the number of inequivalent actions of certain groups of appropriate degrees. These results are all somewhat technical, and so for convenience are all gathered together in this section.

Definition 3.1. We will have need of the wreath product of two groups in this paper, and usually, we will need the "permutational" wreath product, which we now define. Let $G$ 
and $H$ be groups acting on $X$ and $Y$, respectively. We define the wreath product of $X$ and $Y$, denoted $G$ ? $H$, to be the permutation group that acts on $X \times Y$ consisting of all permutations of the form $(x, y) \rightarrow\left(g(x), h_{x}(y)\right)$, where $g \in G$ and $h_{x} \in H$. We remark that the order of $G$ and $H$ is backwards from what is usually used in group theory, but is common in graph theory. We have occasional use of the more abstract definition of the wreath product with its product action. The reader unfamiliar with this action is referred to [5, pg. 50] for this definition as well as basic facts regarding the product action.

Lemma 3.2. Let $p \geq 3$ be prime, and $G$ a primitive group with nonabelian simple socle $T$ of degree $p^{s}, s \geq 1$. Then every transitive representation of $G$ of degree a power of $p$ is of degree $p^{s}$ provided that if $T=\operatorname{PSL}(2,8)$ is of degree 9 , then $G$ contains a cyclic Sylow p-subgroup.

Proof. As $G$ is primitive in its action on $p^{s}$ points, the stabilizer of a point in this action is a subgroup of $G$ of prime-power index $p^{s}$. By [11, Theorem 1], $T$ acts transitively on exactly one prime-power degree, which is necessarily $p^{s}$, and by [11, Corollary 2], this action is primitive. Now suppose that $G$ has a transitive action of degree $p^{t}, t \neq s$. Note that $T$ cannot be transitive in this action, as otherwise the stabilizer of a point in $T$ is a subgroup of index $p^{t}$, but by [11, Theorem 1], we must have that $t=s$. Hence $G$ admits a nontrivial complete block system $\mathcal{C}$ formed by the orbits of $T$. If $t<s$, then $\left.\left.T\right|_{C} \triangleleft \operatorname{fix}_{G}(\mathcal{C})\right|_{C}$ and $\left.T\right|_{C} \cong T$ is transitive on $C \in \mathcal{C}$, and so $\left.T\right|_{C}$ is a transitive group acting on $C$. Then the stabilizer of a point of $\left.T\right|_{C}$ is subgroup of index $p^{t}$, and so by [11, Theorem 1], we must have that $t=s$, a contradiction. If $t>s$, then $G / \mathcal{C}$ has degree a power of $p$, and so $p$ must divide $|G / \mathcal{C}|$. As $T \triangleleft G$, we must have that $G \leq N_{S_{p^{s}}}(T)$. As $G / \mathcal{C}$ is a quotient group of $G / T$, we must then have that $p$ divides $\left|N_{S_{p s}}(T) / T\right|$. By [11, Theorem 1], $T=A_{p^{s}}, T=\operatorname{PSL}(n, q)$ for some prime power $q$ and $n$ a prime with $p^{s}=\left(q^{n}-1\right) /(q-1), T=\operatorname{PSL}(2,11)$ and $p^{s}=11, T=M_{23}$ and $p^{s}=23$, or $T=M_{11}$ and $p^{s}=11$. Note that if $p^{s}$ is prime, then clearly $p$ cannot divide $\left|N_{S_{p^{s}}}(T) / T\right|$, and it is also clear that $T \neq A_{p^{s}}$. The only remaining case is that $T=\operatorname{PSL}(n, q)$, and by [7, Lemma 17] or hypothesis, a Sylow $p$-subgroup of $G$ and $T$ is cyclic, so that $p$ does not divide $\left|N_{S_{p}}(T) / T\right|$, a contradiction.

Lemma 3.3. Let $p \geq 3$ be prime, and let $m=p^{t}$ for some $t \geq 1$. Let $G$ be a primitive nonabelian almost simple group of degree $m$ acting on $\Omega$ with socle $T$, where if $T=$ $\operatorname{PSL}(2,8)$ is of degree 9 , then $G$ contains a cyclic Sylow p-subgroup. Let $K \leq S_{r} \succ G$ be primitive of degree $m^{r}$ acting on $\Omega^{r}$, where $S_{r} \zeta G$ has the product action and $\operatorname{soc}(K)=T^{r}$. Then the number of primitive inequivalent actions of $K$ of degree $m^{r}$ on $\Omega^{r}$ is the number of inequivalent actions of $T$ of degree $m$ acting on $\Omega$, and this number is either 1 or 2 .

Proof. Let $\mathcal{X}$ be the set of all subgroups $H$ of $K$ of index $m^{r}$ in $K$ such that the action of $K$ on the left cosets of $H$ is primitive of degree $m^{r}$, and $\mathcal{Y}$ be the set of all subgroups of $T$ of index $m$ in $R$. Define $f: \mathcal{X} \rightarrow \mathcal{Y}$ by letting $f(X)$ be the projection in the first coordinate of $X \cap T^{r}$. We first show that $f$ is well-defined by showing that $f(X)$ is indeed a subgroup of $T$ of index $m$.

Note that we may view $X$ as the stabilizer of a point in a primitive transitive action of $K$ of degree $m^{r}$ in which case $T^{r}$ is transitive as $T^{r} \triangleleft K$ and a normal subgroup of a primitive group is transitive [19, Theorem 8.8]. By [5, Exercise 1.4.1], we have that $X T^{r}=K$. Then by [12, Theorem 1.4.8], we have that $\left[T^{r}: X \cap T^{r}\right]=[K: X]=m^{r}$. Then the index of the projection of $X \cap T^{r}$ in the first coordinate, call it $L$, has index dividing $m^{r}$ in 
$T$. As $m=p^{t}$ is a prime power, we have that $[T: L]=p^{s}$ for some $s \geq 1$. We conclude that $T$ has a transitive representation of degree $p^{s}$, and by Lemma 3.2, we have that $s=t$. Thus $f(X)$ is a subgroup of $T$ of index $m=p^{t}$ and so $f$ is well defined.

Let $\Omega^{r}=\Pi_{i=1}^{r} \Omega_{i}$, where if $\left(\omega_{1}, \ldots, \omega_{r}\right) \in \Omega^{r}$, then $\omega_{i} \in \Omega_{i}$. We write $T^{r}=\prod_{i=1}^{r} T_{i}$, where each $T_{i}$ is the action of $T$ on $\Omega_{i}$ in $T^{r}$. As $K \leq S_{r}$ 々 $G$ has the product action, the action of $T_{i}$ on $\Omega_{i}$ is the natural action, and so the stabilizer in $T_{i}$ of a point in $\Omega_{i}$ is the stabilizer in $T_{j}$ of a point in $\Omega_{j}$. Thus the action of $T_{i}$ on $\Omega_{i}$ is equivalent to the action of $T_{j}$ on $\Omega_{j}$. Thus if $L_{i, \omega}$ is the stabilizer in $T_{i}$ of the point $\omega$, then $L_{i, \omega}$ is a conjugate in $T$ of $L_{j, \omega^{\prime}}$ for every $1 \leq i, j \leq r, \omega \in \Omega_{i}$ and $\omega^{\prime} \in \Omega_{j}$. Then the stabilizer of the point $\left(\omega_{1}, \ldots, \omega_{r}\right)$ in $T^{r}$ is $\Pi_{i=1}^{r} L_{i, \omega_{i}}$. As $T^{r} \triangleleft K$, we conclude that for any $k \in K$, $k^{-1} L_{i, \omega} k=L_{j, \omega^{\prime}}$, where again $1 \leq i, j \leq r, \omega \in \Omega_{i}$, and $\omega^{\prime} \in \Omega_{j}$.

Now let $k \in K$ such that $k T^{r} \neq T^{r}$, and $\left(\omega_{1}, \ldots, \omega_{r}\right)=\omega \in \Omega^{r}$. As $T^{r}$ is transitive, there exists $h \in T^{r}$ such that $k h$ stabilizes $\omega$. Then $k h T^{r}=k T^{r}$ and $k h$ normalizes $\Pi_{i=1}^{r} L_{i, \omega_{i}}$, the stabilizer of $\omega \in T^{r}$. Thus $\left|N_{K}\left(\Pi_{i=1}^{r} L_{i, \omega_{i}}\right) / \Pi_{i=1}^{r} L_{i, \omega_{i}}\right|=\left|K / T^{r}\right|$. We conclude that $N_{K}\left(\Pi_{i=1}^{r} L_{i, \omega_{i}}\right)$ is a subgroup of index $m^{r}$ in $K$ and so is the stabilizer of a point of $\Omega^{r}$.

Now suppose that $K$ has another primitive representation, which we will call $\rho(K)$, of degree $m^{k}$, that is inequivalent to the natural representation as a subgroup of $S_{r} \prec G$. Let $X$ be the stabilizer of a point in $\rho(K)$, so that $f(X) \leq T$ is a subgroup of index $m$ in $T$. Set $L=L_{1, \omega_{1}}, \omega_{1} \in \Omega_{1}$, where we regard $L$ as a subgroup of $T$ of index $m$. If $f(X)$ is a conjugate of $L$ in $T$, then $f(X)=L_{1, \omega_{1}^{\prime}}$ for some $\omega_{1}^{\prime} \in \Omega_{1}$, and as $k^{-1} L_{i, \omega} k=L_{j, \omega^{\prime}}$ for every $k \in K, 1 \leq i, j \leq r, \omega \in \Omega_{i}$ and some $\omega^{\prime} \in \Omega_{j}$, we have that $\operatorname{Stab}_{\rho(K)}\left(\omega_{1}, \ldots, \omega_{r}\right)=\Pi_{i=1}^{r} L_{i, \omega_{i}}$. Arguing as in the previous paragraph, we have that $N_{K}\left(\Pi_{i=1}^{r} L_{i, \omega_{i}}\right)$ is the stabilizer in $\rho(K)$ of $\left(\omega_{1}, \ldots, \omega_{r}\right)$ and so the representation $\rho(K)$ is the same representation as the natural representation of $K$. We may thus assume without loss of generality that $f(X)$ is not a conjugate of $L$ in $T$. This then implies that $T$ has at least two inequivalent transitive representations of degree $m$, and so if $T$ has exactly one representation up to equivalence, the result follows.

Now, by [11, Corollary 2], we have that $T$ is doubly-transitive. By [3, Table], we have that $T$ has at most two inequivalent representations of degree $m$, and so $T$ has exactly two inequivalent representations of degree $m$. In $\rho(K)$, let $M_{i, \omega_{i}}$ be the stabilizer of the point $\omega_{i} \in \Omega_{i}$ in $T_{i}$. By arguments above, we have that no $M_{i, \omega_{i}}$ is conjugate to any $L_{i, \omega_{j}}, \omega_{j} \in \Omega_{i}$. As there are two inequivalent representations of $T$ of degree $m$, there are exactly two conjugacy classes of subgroups of index $m$ in $T$ and as for any $k \in K$, $k^{-1} L_{i, \omega} k=L_{j, \omega^{\prime}}$, we have that for any $k \in K, k^{-1} M_{i, \omega} k=M_{j, \omega^{\prime}}$. Arguing as above, we conclude that the stabilizer of the point $\left(\omega_{1}, \ldots, \omega_{r}\right) \in \Omega^{r}$ in $\rho(K)$ is $N_{K}\left(\Pi_{i=1}^{r} M_{i, \omega_{i}}\right)$. Arguing as above, we conclude that $K$ has exactly two inequivalent representations if $T$ has two inequivalent representations.

Lemma 3.4. Let $p \geq 3$, and $m=p^{t}$. Let $T$ be a doubly-transitive simple group of degree $m$ acting on $\Omega$. Then the number of inequivalent transitive actions of $T^{r}$ acting coordinatewise on $\Omega^{r}$ is $a^{r}$, where $a$ is the number of inequivalent actions of $T$ on $\Omega$. Consequently, $a^{r}=1$ or $a^{r}=2^{r}$.

Proof. Similarly to Lemma 3.3, we define $\mathcal{X}$ to be the set of all subgroups $H$ of $T^{r}$ of index $m^{r}$ in $T^{r}$ such that the action of $T^{r}$ on the left cosets of $H$ is transitive of degree $m^{r}$, and $\mathcal{Y}$ be the set of all subgroups of $T$ of index $m$ in $T$. Define $f_{i}: \mathcal{X} \rightarrow \mathcal{Y}$ by letting $f_{i}(X)$ be the projection in the $i^{t h}$-coordinate of $X \cap T^{r}$. Using the argument in Lemma 3.3 
to show that $f$ is well-defined, we have that each $f_{i}$ is well-defined. As there are $a$ choices for each $f_{i}(X)$ and there are $r$ coordinates, we have that $|\mathcal{X}|=a^{r}$. It then follows by [3, Table] that $a=1$ or 2 .

Lemma 3.5. Let $G \leq S_{p^{k}}$, $p$ a prime and $k \geq 2$, be transitive with abelian Sylow $p$ subgroup $P$ and admit a normal complete block system $\mathcal{B}$ such that $\left.\operatorname{Stab}_{G}(B)\right|_{B}$ is primitive. Suppose that $\operatorname{fix}_{G}(\mathcal{B})$ contains a characteristic subgroup $K$ whose orbits form $\mathcal{B},\left.K\right|_{B}$ is equivalent to $\left.K\right|_{B^{\prime}}$ for every $B, B^{\prime} \in \mathcal{B}$, and $\left.K\right|_{B}$ is imprimitive. Then $\left.\operatorname{Stab}_{P^{G}}(B)\right|_{B}$ is imprimitive.

Proof. Let $P_{1}$ be a Sylow $p$-subgroup of $\operatorname{fix}_{G}(\mathcal{B})$ that is contained in $P$. Note that $P_{1}$ is necessarily semiregular and abelian. As $K$ is characteristic and Sylow $p$-subgroups are conjugate, every Sylow $p$-subgroup of $\operatorname{fix}_{G}(\mathcal{B})$ is contained in $K$. Let $\mathcal{B}$ consist of $p^{\ell}$ blocks of size $p^{m}$. We consider $G$ as acting on $\mathbb{Z}_{p^{\ell}} \times\left(P_{1}\right)_{L}$ so that $\mathcal{B}=\{\{(i, j): j \in$ $\left.\left.P_{1}\right\}: i \in \mathbb{Z}_{p^{\ell}}\right\}$. Then $g(i, j)=\left(\sigma_{g}(i), \omega_{g, i}(j)\right)$, for $g \in G$, where $\sigma_{g} \in S_{p^{\ell}}$ and each $\omega_{g, i} \in S_{P_{1}}$. We may also assume that if $\rho \in P_{1}$, then $\rho(i, j)=(i, j+b)$, where $b \in P_{1}$. As $\left.K\right|_{B}$ is equivalent to $\left.K\right|_{B^{\prime}}$ for every $B, B^{\prime} \in \mathcal{B}$, we may additionally assume that if $k \in K$, then $k(i, j)=(i, \kappa(j))$, where $\kappa \in S_{P_{1}}$. Let $g \in G$. Then $g^{-1} P_{1} g \leq K$ is a Sylow $p$-subgroup of $K$ (and $\operatorname{fix}_{G}(\mathcal{B})$ ), and so there exists $k_{g} \in K$ such that $k_{g}^{-1} g^{-1} P_{1} g k_{g}=P_{1}$. Then $g k_{g}$ normalizes $P_{1}$, and $N_{S_{\mathbb{Z}_{p} \ell\left(P_{1}\right)_{L}}}\left(P_{1}\right)=\left\{\left(\sigma(i), \alpha(j)+b_{i}\right): \sigma \in S_{p^{\ell}}, \alpha \in\right.$ $\left.\operatorname{Aut}\left(P_{1}\right), b_{i} \in P_{1}\right\}$. Thus $g k_{g}(i, j)=\left(\sigma(i), \alpha(j)+b_{i}\right)$ for appropriate $\sigma, \alpha$, and $b_{i}$ (note that $\left.\left(g k_{g}\right)^{-1}(i, j)=\left(\sigma^{-1}(i), \alpha^{-1}(j)-\alpha^{-1}\left(b_{\sigma^{-1}(i)}\right)\right)\right)$. Also, $k_{g}(i, j)=\left(i, \omega_{k_{g}, i}(j)\right)$, so as $k_{g} \in K$, we may set $k_{g}(i, j)=(i, \omega(j)), \omega \in S_{P_{1}}$. As $P$ is transitive and abelian, if $\beta \in P$, then $\beta(i, j)=\left(\sigma_{\beta}(i), j+c_{i}\right), c_{i} \in P_{1}$. Then

$$
\begin{aligned}
g^{-1} \beta g(i, j) & =k_{g}\left(k_{g}^{-1} g^{-1}\right) \beta\left(g k_{g}\right) k_{g}^{-1}(i, j) \\
& =\left(\sigma^{-1} \sigma_{\beta} \sigma(i), \omega\left(\omega^{-1}(j)+\alpha^{-1}\left(b_{i}\right)+\alpha^{-1}\left(c_{\sigma(i)}\right)-\alpha^{-1}\left(b_{\sigma^{-1} \sigma_{\beta} \sigma(i)}\right)\right)\right) .
\end{aligned}
$$

As each map $(i, j) \rightarrow\left(i, j+d_{i}\right), d_{i} \in P_{1}$, is contained in $\left.\operatorname{fix}_{P_{1}}(\mathcal{B})\right|_{B} \leq\left. K\right|_{B}, B \in$ $\mathcal{B}$, and $\omega,\left.\omega^{-1} \in K\right|_{B}, B \in \mathcal{B}$ we conclude that $P^{G} \leq(G / \mathcal{B}) \imath\left(\left.K\right|_{B}\right)$ for $B \in \mathcal{B}$. Then $\left.\operatorname{Stab}_{P G}(B)\right|_{B} \leq\left. K\right|_{B}$ which is imprimitive, and the result follows by [5, Exercise 1.5.10].

The following result is ultimately where the requirement that $p>2^{t-1}$ originates. A deeper analysis of the number of inequivalent actions of $\operatorname{fix}_{G}(\mathcal{B})$ in the following result could lead to the removal of the condition that $p>2^{t-1}$.

Lemma 3.6. Let $p \geq 3$ be prime, and let $G \leq S_{p^{k}}$ be transitive such that $G$ contains an abelian Sylow p-subgroup $P$ and $G$ admits a complete block system $\mathcal{B}$ such that $\left.\operatorname{Stab}_{G}(\mathcal{B})\right|_{B}$ is primitive and not permutation isomorphic to a subgroup of $\operatorname{AGL}(r, p)$ for any $r \geq 1$, but $\left.\operatorname{fix}_{G}(\mathcal{B})\right|_{B}$ is imprimitive, $B \in \mathcal{B}$. Let $t$ be the rank of a Sylow p-subgroup of fix $_{G}(\mathcal{B})$. If $p>2^{t}$, then $P^{G}$ admits a nontrivial complete block system $\mathcal{C}$ such that $\mathcal{C} \prec \mathcal{B}$.

Proof. As $\left.\operatorname{Stab}_{G}(B)\right|_{B}$ is primitive, $\left.\operatorname{Stab}_{G}(B)\right|_{B}$ is given by the O'Nan-Scott Theorem (we use the form given by [17]). As $\left.\operatorname{Stab}_{G}(B)\right|_{B}$ is a primitive group of odd prime-power degree, $B \in \mathcal{B}$, we have that $\left.\operatorname{Stab}_{G}(B)\right|_{B}$ is a subgroup of $S_{m} \prec K$ with the product action, where $K$ is an almost simple group of degree a power of $p$ as $\left.\operatorname{Stab}_{G}(B)\right|_{B}$ is not permutation isomorphic to a subgroup of $\operatorname{AGL}(r, p)$. Note that $\left.\operatorname{Stab}_{G}(B)\right|_{B}$ cannot 
be an almost simple group as $\left.\operatorname{fix}_{G}(\mathcal{B})\right|_{B}$ is imprimitive and normal in $\left.\operatorname{Stab}_{G}(B)\right|_{B}$. By [11, Theorem 1], we have that $K$ is doubly-transitive. Then $\operatorname{soc}\left(\left.\operatorname{fix}_{G}(\mathcal{B})\right|_{B}\right)=T^{r}$ acting coordinate-wise on $\Omega^{r}$ for some doubly-transitive simple group $T$ of degree $p^{k / r}$ acting on $\Omega$. Also, $\operatorname{soc}\left(\left.\operatorname{fix}_{G}(\mathcal{B})\right|_{B}\right)$ is characteristic in $\left.\operatorname{Stab}_{G}(B)\right|_{B}$, and so normal, and as a normal subgroup of a primitive group is transitive [19, Theorem 8.8], we have that $T^{r}$ is transitive as well.

Observe that $T^{r}$ must act faithfully on each $B \in \mathcal{B}$, as otherwise $T^{r}$ contains a normal subgroup $H$ that is nontrivial (and so has orbits of size dividing a multiple of $p$ ) on some block $B \in \mathcal{B}$ while $H$ acts trivially on some $B^{\prime} \in \mathcal{B}$. Then $H$ contains a nontrivial Sylow $p$ subgroup which necessarily fixes a point, while $P$ is regular. Define an equivalence relation $\equiv$ on $\mathcal{B}$ by $B \equiv B^{\prime}$ if and only if the action of $T^{r}$ on $B$ is equivalent to the action of $T^{r}$ on $B^{\prime}$. By [5, Lemma 1.6B], $B \equiv B^{\prime}$ if and only if there exists $x \in B$ and $x^{\prime} \in B^{\prime}$ such that $\operatorname{Stab}_{\left.T^{r}\right|_{B}}(x)=\operatorname{Stab}_{\left.T^{r}\right|_{B^{\prime}}}\left(x^{\prime}\right)$. As conjugation by an element of $G$ maps the stabilizer of some point of $\left.T^{r}\right|_{B}$ to the stabilizer of some point of $\left.T^{r}\right|_{B^{\prime}}$ for some $B^{\prime} \in \mathcal{B}$, we see that if $g \in G$, then $g(B) \equiv g\left(B^{\prime}\right)$. As $G / \mathcal{B}$ permutes the blocks of $\mathcal{B}$, we see that $\equiv$ is a $G / \mathcal{B}$ congruence, and so by [5, Exercise 1.5.4], the equivalence classes of $\equiv$ are blocks of $G / \mathcal{B}$. By Lemma 3.4 we have that $T^{r}$ has at most $2^{r} \leq 2^{t}<p$ inequivalent representations. We conclude that there is exactly one equivalence class of $\equiv$, and by $\left.\operatorname{Lemma} 3.5 \operatorname{Stab}_{P^{G}}(\mathcal{B})\right|_{B}$ is imprimitive. The result then follows by [5, Exercise 1.5.10].

Lemma 3.7. Let $H \leq \operatorname{AGL}(k, p)$ be transitive such that a Sylow p-subgroup of $H$ is elementary abelian of order $p^{k}$. Then $H$ has a unique action of degree $p^{k}$.

Proof. As $H \leq \mathrm{AGL}(k, p)$ is transitive, $H$ contains a regular normal elementary abelian Sylow $p$-subgroup $P$ of order $p^{k}$. As $P$ is solvable, by the Schur-Zassenhaus Theorem, $P$ contains a complement $K$ of order $|H| / p^{k}$, and any two subgroups of $H$ of order $|H| / p^{k}$ are conjugate in $H$. We conclude by [5, Lemma 1.6B] and the comments following it that $H$ has a unique action of degree $p^{k}$.

\section{Direct Products}

In this section, we have gathered together all of the results that consider when $\operatorname{fix}_{G}(\mathcal{B})$ is not semiregular and conclude with $G$ being contained in a direct product. Our main tool is the following result.

Lemma 4.1. Let $G \leq S_{n}$ be transitive such that $G$ admits a normal complete block system $\mathcal{B}$ consisting of $m$ blocks of size $k$. If $\operatorname{fix}_{G}(\mathcal{B})$ acts faithfully on $B \in \mathcal{B}$, $\left.\operatorname{fix}_{G}(\mathcal{B})\right|_{B}$ is equivalent to $\left.\operatorname{fix}_{G}(\mathcal{B})\right|_{B^{\prime}}$ for every $B, B^{\prime} \in \mathcal{B}$, and the stabilizer of a point in $\left.\operatorname{fix}_{G}(\mathcal{B})\right|_{B}$ fixes exactly one point for $B \in \mathcal{B}$, then $G$ is permutation isomorphic to subgroup of $S_{m} \times S_{k}$ with the canonical action.

Proof. We assume without loss of generality that $G$ acts on $\mathbb{Z}_{m} \times \mathbb{Z}_{k}$ so that $\mathcal{B}=\{\{(i, j)$ : $\left.\left.j \in \mathbb{Z}_{p^{k}}\right\}: i \in \mathbb{Z}_{m}\right\}$. Define an equivalence relation $\equiv$ on $\mathbb{Z}_{m} \times \mathbb{Z}_{k}$ by $(i, j) \equiv\left(i^{\prime}, j^{\prime}\right)$ if and only if $\operatorname{Stab}_{\operatorname{fix}_{G}(\mathcal{B})}(i, j)=\operatorname{Stab}_{\mathrm{fix}_{G}(\mathcal{B})}\left(i^{\prime}, j^{\prime}\right)$. It is easy to see that the equivalence classes of $\equiv$ form a complete block system $\mathcal{E}$ of $H$. If $\left.\operatorname{fix}_{G}(\mathcal{B})\right|_{B}$ has only one action up to equivalence and the stabilizer of a point in $\left.\operatorname{fix}_{G}(\mathcal{B})\right|_{B}$ fixes only one point, then $\mathcal{E}$ consists of $k$ blocks of size $m$, and each block of $\mathcal{E}$ contains exactly element of each block in $\mathcal{B}$ by [5, Lemma 1.6B]. The result then follows by [6, Lemma 2.2]. 
Lemma 4.2. Let $G \leq S_{n}$, nodd, such that $G$ admits a complete block system $\mathcal{B}$ consisting of $m$ blocks of size $p^{k}, p$ a prime and $k \geq 1$, and one of the following is true:

1. a Sylow p-subgroup of $\left.\operatorname{fix}_{G}(\mathcal{B})\right|_{B}$ contains a regular cyclic subgroup and $\left.\operatorname{fix}_{G}(\mathcal{B})\right|_{B}$ is a primitive group with nonabelian socle, or

2. a Sylow p-subgroup of $\operatorname{fix}_{G}(\mathcal{B})$ is cyclic and semiregular of order $p^{k}$.

Then one of the following is true:

i. $\operatorname{fix}_{G}(\mathcal{B})$ is cyclic and semiregular of order $p^{k}$,

ii. $G$ is permutation isomorphic to a subgroup of $S_{m} \times S_{p^{k}}$ with the canonical action. Furthermore, if $G$ contains a regular abelian group, then there exists $H \leq S_{m}$ and $K \leq S_{p^{k}}$ such that $H \times K \triangleleft G$, or

iii. $\operatorname{fix}_{G}(\mathcal{B})$ does not act faithfully on $B \in \mathcal{B}$ and a Sylow p-subgroup of $\operatorname{fix}_{G}(\mathcal{B})$ is not semiregular.

Proof. We assume without loss of generality that $G$ acts on $\mathbb{Z}_{m} \times \mathbb{Z}_{p^{k}}$ so that $\mathcal{B}=\{\{(i, j)$ : $\left.\left.j \in \mathbb{Z}_{p^{k}}\right\}: i \in \mathbb{Z}_{m}\right\}$. Note that if $\operatorname{fix}_{G}(\mathcal{B})$ does not act faithfully on $B \in \mathcal{B}$, then there exists some normal subgroup $N \triangleleft \operatorname{fix}_{G}(\mathcal{B})$ and $B, B^{\prime} \in \mathcal{B}$ such that $\left.N\right|_{B}=1$ while $\left.N\right|_{B^{\prime}} \neq 1$. As $\left|B^{\prime}\right|=p^{k}$, the orbits of $\left.N\right|_{B^{\prime}}$ have order a power of $p$, and so $p$ divides $|N|$. Thus $N$ contains an element of order $p$ that fixes a point, and so if $\operatorname{fix}_{G}(\mathcal{B})$ does not act faithfully on $B \in \mathcal{B}$, then a Sylow $p$-subgroup of $\operatorname{fix}_{G}(\mathcal{B})$ is not semiregular. We may now assume without loss of generality that $\operatorname{fix}_{G}(\mathcal{B})$ acts faithfully on $B \in \mathcal{B}$, as otherwise (iii) occurs. We may also assume that $\operatorname{fix}_{G}(\mathcal{B})$ is not cyclic and semiregular of order $p^{k}$ as then (i) occurs.

First suppose that $\left.\operatorname{fix}_{G}(\mathcal{B})\right|_{B}$ is primitive with nonabelian socle. If $k=1$, then by Burnside's Theorem [5, Theorem 3.5B], we have that $\operatorname{fix}_{G}(\mathcal{B})$ is doubly-transitive. If $k \geq 2$, then as $\mathbb{Z}_{p^{k}}$ is a Burnside group [5, Theorem 3.5A], we also have that $\left.\operatorname{fix}_{G}(\mathcal{B})\right|_{B}$ is doubly-transitive. Thus in any case, $\left.\operatorname{fix}_{G}(\mathcal{B})\right|_{B}$ is doubly-transitive with nonabelian socle. Define an equivalence relation $\equiv$ on $\mathbb{Z}_{m} \times \mathbb{Z}_{p^{k}}$ by $(i, j) \equiv\left(i^{\prime}, j^{\prime}\right)$ if and only if $\operatorname{Stab}_{\text {fix }_{G}(\mathcal{B})}((i, j))=\operatorname{Stab}_{\text {fix }_{G}(\mathcal{B})}\left(\left(i^{\prime}, j^{\prime}\right)\right)$. It is easy to see that the equivalence classes of $\equiv$ are blocks of $G$, forming the complete block system $\mathcal{E}$. Now, $\left.f_{i x}(\mathcal{B})\right|_{B}$ is one of the groups given in [16, Theorem 1.1], and by [3, Theorem 5.3, Table], each of these groups has either one or two inequivalent actions. As $\left.\operatorname{fix}_{G}(\mathcal{B})\right|_{B}$ is doubly-transitive, we have that the stabilizer of a point in $\left.\operatorname{fix}_{G}(\mathcal{B})\right|_{B}$ fixes exactly one point, $B \in \mathcal{B}$. The first statement in (ii) then follows by Lemma 4.1 if $\left.\operatorname{fix}_{G}(\mathcal{B})\right|_{B}$ is equivalent to $\left.\operatorname{fix}_{G}(\mathcal{B})\right|_{B^{\prime}}$ for every $B, B^{\prime} \in \mathcal{B}$. Otherwise, $\left.\operatorname{fix}_{G}(\mathcal{B})\right|_{B}$ must have exactly two inequivalent actions, and $\mathcal{E}$ must consist of an even number of blocks which is impossible as $m$ is odd. Thus the first statement of (ii) follows.

If $\left.\operatorname{fix}_{G}(\mathcal{B})\right|_{B}$ is not primitive with nonabelian socle, then $\operatorname{fix}_{G}(\mathcal{B})$ contains a cyclic and semiregular Sylow $p$-subgroup. Then $\left.\operatorname{fix}_{G}(\mathcal{B})\right|_{B}$ contains a cyclic and transitive Sylow $p$ subgroup. By Theorem $\left.1.2 \operatorname{fix}_{G}(\mathcal{B})\right|_{B}$ contains a normal cyclic Sylow $p$-subgroup. Thus $\left.\operatorname{fix}_{G}(\mathcal{B})\right|_{B} \leq \mathbb{Z}_{p^{k}}^{*} \cdot\left(\mathbb{Z}_{p^{k}}\right)_{L}$, and $\mathbb{Z}_{p^{k}}^{*}$ has order $p^{k-1}(p-1)$, and is solvable. We conclude that $\left.\operatorname{fix}_{G}(\mathcal{B})\right|_{B}$ has order dividing $(p-1) p^{k}$. Let $\left|\operatorname{fix}_{G}(\mathcal{B})\right|_{B} \mid=a \cdot p^{k}$, so that $\operatorname{gcd}(a, p)=$ 1. Note that $\operatorname{fix}_{G}(\mathcal{B})=\langle\rho\rangle$ and is semiregular if $a=1$, and the result follows. We thus assume that $a \neq 1$. As $\left.\operatorname{fix}_{G}(\mathcal{B})\right|_{B}$ is solvable, $\left.\operatorname{fix}_{G}(\mathcal{B})\right|_{B}$ contains a subgroup $A$ of order $a$, and by Hall's Theorem [12, Proposition II.7.14], any two subgroups of $\left.\operatorname{fix}_{G}(\mathcal{B})\right|_{B}$ of order $a$ are conjugate in $\left.\operatorname{fix}_{G}(\mathcal{B})\right|_{B}$. As any action of $\left.\operatorname{fix}_{G}(\mathcal{B})\right|_{B}$ on $p^{k}$ points can be 
viewed as the action of $\left.\operatorname{fix}_{G}(\mathcal{B})\right|_{B}$ on the right cosets of some subgroup $A^{\prime}$ of order $a$, and all such subgroups are conjugate, by [5, Lemma 1.6A] and the comments following it, $\left.\operatorname{fix}_{G}(\mathcal{B})\right|_{B}$ has a unique action as well. Note that $c x \equiv x\left(\bmod p^{k}\right)$ is equivalent to $(c-1) x \equiv 0\left(\bmod p^{k}\right)$. This last equation will have a unique solution provided that $c-1 \not \equiv 0(\bmod p)$. That is $c x \equiv x\left(\bmod p^{k}\right)$ has a unique solution unless $c=1+b p$ for some $b$. As $1+b p$ has multiplicative order a power of $p$ in $\mathbb{Z}_{p^{k}}$ for every $b$ and $\left.\operatorname{fix}_{H}(\mathcal{B})\right|_{B} \leq$ $N_{S_{p^{k}}}\left(\left(\mathbb{Z}_{p^{k}}\right)_{L}\right)=\left\{x \rightarrow a_{1} x+b_{1}: a_{1} \in \mathbb{Z}_{p^{k}}^{*}, b_{1} \in \mathbb{Z}_{p^{k}}\right\}$, we conclude that the stabilizer of a point in $\left.\operatorname{fix}_{G}(\mathcal{B})\right|_{B}$ fixes exactly one point. Then $E \cap B$ is a singleton for every $E \in \mathcal{E}$ and $B \in \mathcal{B}$, and the first statement of (ii) follows by Lemma 4.1. Thus in any case, the first statement of (ii) follows.

If in addition $G$ contains a regular abelian group, set $H=\operatorname{fix}_{G}(\mathcal{E}) \neq 1$ and $K=$ $\operatorname{fix}_{G}(\mathcal{B})$. Then the internal direct product $H \times K \triangleleft G$ and is transitive. Hence (ii) follows.

Lemma 4.3. Let $G \leq S_{n}$, n odd, admit a complete block system $\mathcal{B}$ of $n / p^{k}$ blocks of size $p^{k}$ such that a Sylow p-subgroup of $\operatorname{fix}_{G}(\mathcal{B})$ is a semiregular direct product of cyclic groups of prime-power order. If $\left.\operatorname{fix}_{G}(\mathcal{B})\right|_{B}$ is primitive, then $G$ is permutation isomorphic to a subgroup of $S_{n / p^{k}} \times S_{p^{k}}$.

Proof. As $\left.\operatorname{fix}_{G}(\mathcal{B})\right|_{B}$ is primitive and a Sylow $p$-subgroup of $\operatorname{fix}_{G}(\mathcal{B})$ is a semiregular direct product of cyclic groups of prime-power order, we have a Sylow $p$-subgroup of $\left.\operatorname{fix}_{G}(\mathcal{B})\right|_{B}$ is direct product of cyclic groups of prime-power order. It follows by [16, Theorem 1.1] that $\left.\operatorname{fix}_{G}(\mathcal{B})\right|_{B}$ is permutation isomorphic to a subgroup of $\operatorname{AGL}(k, p)$ or $S_{r} \prec K$, where $K$ is primitive group of degree $p^{k / r}$ with nonabelian socle (note that the possible socles are given in [16, Corollary 1.2] as $K$ contains a regular cyclic subgroup). Observe that $\operatorname{fix}_{G}(\mathcal{B})$ must act faithfully on $B \in \mathcal{B}$, as otherwise $\operatorname{fix}_{G}(\mathcal{B})$ contains a normal subgroup $N$ which is trivial on $B$ but nontrivial on some $B^{\prime} \in \mathcal{B}$. If this occurs, we have that $p^{k}$ divides $|N|_{B^{\prime}} \mid$ as a normal subgroup of a primitive group is transitive, and so a Sylow $p$-subgroup of $\operatorname{fix}_{G}(\mathcal{B})$ has order at least $p^{2 k}$, which does not occur. If $\left.\operatorname{fix}_{G}(\mathcal{B})\right|_{B}$ is permutation isomorphic to a subgroup of $\operatorname{AGL}(k, p)$, then by Lemma 3.7, we have that $\left.\operatorname{fix}_{G}(\mathcal{B})\right|_{B}$ has a unique action of degree $p^{k}$, and as $\left.\operatorname{fix}_{G}(\mathcal{B})\right|_{B}$ is primitive, the stabilizer of a point in $\left.\operatorname{fix}_{G}(\mathcal{B})\right|_{B}$ must fix exactly one point. The result then follows by Lemma 4.1.

If $\left.\operatorname{fix}_{G}(\mathcal{B})\right|_{B}$ is permutation isomorphic to a subgroup of $S_{r} \prec K$, then by Lemma 3.3, the number of inequivalent actions of $\left.\operatorname{fix}_{G}(\mathcal{B})\right|_{B}$ of degree $p^{k}$ is the number of inequivalent actions of $K$ of degree $p^{k / r}$, and this number of inequivalent actions is either 1 or 2 . If $K$ has one action of degree $p^{k / r}$, then the result follows by Lemma 4.1. If $K$ has two actions of degree $p^{k / r}$, then define $\equiv$ as in Lemma 4.1. If for some $B^{\prime} \in \mathcal{B}$ we have that $\left.\operatorname{fix}_{G}(\mathcal{B})\right|_{B}$ is inequivalent to $\left.\operatorname{fix}_{G}(\mathcal{B})\right|_{B^{\prime}}$, then the equivalence classes of $\equiv$ have even order, and so $G$ admits a complete block system whose blocks have an even number of points. This, however, is not possible as $n$ is odd. Thus $\left.\operatorname{fix}_{G}(\mathcal{B})\right|_{B}$ is equivalent to $\left.\operatorname{fix}_{G}(\mathcal{B})\right|_{B^{\prime}}$ for every $B, B^{\prime} \in \mathcal{B}$, and the result follows by Lemma 4.1 .

\section{$5 \operatorname{fix}_{G}(\mathcal{B})$ is semiregular}

In this section, we consider the case when $\operatorname{fix}_{G}(\mathcal{B})$ is semiregular.

Lemma 5.1. Let $G \leq S_{p^{k}}, k \geq 2$ such that $G$ contains a regular abelian p-subgroup $P$ and admits a nontrivial complete block system $\mathcal{B}$ such that $\left.\operatorname{Stab}_{G}(B)\right|_{B}$ normalizes $\left.P\right|_{B}$ for every $B \in \mathcal{B}$, which always occurs if $\operatorname{fix}_{G}(\mathcal{B})$ is semiregular. Then $\operatorname{fix}_{P}(\mathcal{B})$ is contained in 
the center of $P^{G}$, the normal closure of $P$ in $G$, and $P^{G} \leq S_{p^{k-j}} \nmid K$, where $K \cong$ fix $P(\mathcal{B})$ has order $p^{j}$.

Proof. As a transitive abelian group is regular [19, Proposition 4.4] and the homomorphic image of an abelian group is abelian, we have that $\mathcal{B}$ is formed by the orbits of a subgroup $K$ of $P$ of order $p^{j}$. Let $L=P / K$, so that $L$ has order $p^{i}$ with $i+j=k$. We view $G$ as acting on $L \times K$ (even if $P \nsubseteq L \times K$ ) in such a way that $\mathcal{B}$ is formed by the orbits of $1_{L} \times K$. We also assume without loss of generality that $K=K_{L}$, the left regular representation of $K$. Let $g \in G$. Then $g(\ell, k)=\left(\sigma(\ell), \beta_{\ell}(k)\right)$, where $\sigma \in S_{L}$ and each $\beta_{\ell} \in S_{K}$. As $\left.\operatorname{Stab}_{G}(\mathcal{B})\right|_{B}$ normalizes $K_{L}$, by [5, Corollary 4.2B] we have that $\left.\operatorname{Stab}_{G}(\mathcal{B})\right|_{B}$ is isomorphic to a subgroup of $\operatorname{Aut}(K) \cdot K$. Thus $\beta_{\ell}(k)=\alpha_{\ell}(k)+b_{\ell}$, where $\alpha_{\ell} \in \operatorname{Aut}(K)$ and each $b_{\ell} \in K$. A straightforward computation will show that $g^{-1}(\ell, k)=\left(\sigma^{-1}(\ell), \alpha_{\sigma^{-1}(\ell)}^{-1}(k)-\alpha_{\sigma^{-1}(\ell)}^{-1}\left(b_{\ell}\right)\right)$. Let $b \in K$ and $\rho(k, \ell)=(k, \ell+b)$. An equally straightforward computation will show that $g^{-1} \rho g(\ell, k)=\left(\ell, k+\alpha_{\ell}^{-1}(b)\right)$. As $g^{-1} \rho g \in \operatorname{fix}_{G}(\mathcal{B})$, we must have that $\alpha_{\ell}^{-1}(b)=\alpha_{m}^{-1}(b)$ for every $\ell, m \in L$. As this must hold for every $b \in K$, we conclude that $\alpha_{\ell}=\alpha_{m}$ for every $\ell, m \in L$. Hence $g(\ell, k)=\left(\sigma(\ell), \alpha(k)+b_{\ell}\right)$, where $\sigma \in S_{L}, \alpha \in \operatorname{Aut}(K)$, and each $b_{\ell} \in K$. As if $g \in G$, then $g(\ell, k)=\left(\sigma(\ell), \alpha(k)+b_{\ell}\right)$ as above, if $h \in P^{G}$, then $h(\ell, k)=\left(\delta(\ell), k+c_{\ell}\right)$, where $\delta \in S_{L}$ and each $c_{\ell} \in K$. As for every $\rho \in \operatorname{fix}_{G}(\mathcal{B}), \rho(\ell, k)=(\ell, k+b), b \in K$, it is easy to see that every element of $K$ commutes with every elements of $P^{G}$, so that $K \leq Z\left(P^{G}\right)$, the center of $P^{G}$. It is equally straightforward to see that $P^{G} \leq S_{p^{k-j}} \succ K$ as well.

Definition 5.2. We denote the commutator subgroup of $G$ by $G^{\prime}$ and the center of $G$ by $Z(G)$.

Lemma 5.3. Let $G \leq S_{p^{k}}$, $p$ an odd prime and $k \geq 2$, be transitive with Sylow p-subgroup $P$ that is abelian. Suppose that $G$ admits a complete block system $\mathcal{B}$ such that $\operatorname{fix}_{G}(\mathcal{B}) \leq$ $Z(G)$, and $G / \mathcal{B}$ is nonabelian. Then $G^{\prime} \cap \operatorname{fix}_{G}(\mathcal{B})=1, G^{\prime} \neq 1$, and $G^{\prime} / \mathcal{B} \neq 1$.

Proof. Observe first that $\operatorname{fix}_{G}(\mathcal{B})$ is semiregular as $\operatorname{fix}_{G}(\mathcal{B}) \leq Z(G)$ and $Z(G) \leq P$ as an abelian group is self-centralizing [5, Theorem 4.2A (v)]. Note that $P^{\prime}$, the commutator subgroup of $P$, is trivial as $P$ is abelian, and as $P$ is a Sylow $p$-subgroup of $G, P$ is a Hall subgroup of $G$. By [13, Satz IV.2.2], we have that $P \cap G^{\prime} \cap Z(G)=1$. As $G / \mathcal{B}$ is nonabelian, $G^{\prime} \neq 1 \neq G^{\prime} / \mathcal{B}$. As $\operatorname{fix}_{G}(\mathcal{B}) \leq Z(G)$ and a regular abelian group is selfcentralizing, we have that $\operatorname{fix}_{G}(\mathcal{B}) \leq P$. Also, as $\operatorname{fix}_{G}(\mathcal{B}) \leq P \cap Z(G)$, we have that $G^{\prime} \cap \operatorname{fix}_{G}(\mathcal{B})=1$.

Lemma 5.4. Let $G \leq S_{n}$ contain a regular abelian subgroup. If $\mathcal{B}$ and $\mathcal{C}$ are complete block systems of $G$ such that $\operatorname{fix}_{G}(\mathcal{B}) \cap \operatorname{fix}_{G}(\mathcal{C})=1$, then $|B \cap C| \leq 1$ for every $B \in \mathcal{B}$, $C \in \mathcal{C}$, and $\operatorname{fix}_{G}(\mathcal{C})$ acts faithfully on $\mathcal{B}$.

Proof. As $G$ contains a regular abelian subgroup, say $R, \mathcal{B}$ and $\mathcal{C}$ are formed by the orbits of subgroups of $R$, say, $S$ and $T$, respectively. As $\operatorname{fix}_{G}(\mathcal{B}) \cap \operatorname{fix}_{G}(\mathcal{C})=1$, we have that $S \cap T=1$. As the intersection of blocks is again a block, if $B \in \mathcal{B}$ and $C \in \mathcal{C}$ such that $B \cap C \neq \emptyset$, then $B \cap C$ is a block of $G$, and so is an orbit of a subgroup $U$ of $R$. Then $U \leq S \cap T$ so that $U=1$. Thus $|B \cap C| \leq 1$ for every $B \in \mathcal{B}$ and $C \in \mathcal{C}$. Now suppose that $\operatorname{fix}_{G}(\mathcal{C})$ does not act faithfully on $\mathcal{B}$. Then there exists nontrivial $H \leq \operatorname{fix}_{G}(\mathcal{C})$ such that $h(B)=B$ for every $B \in \mathcal{B}$, and so $H \leq \operatorname{fix}_{G}(\mathcal{B})$. As $\operatorname{fix}_{G}(\mathcal{B}) \cap \operatorname{fix}_{G}(\mathcal{C})=1$, we have that $H=1$, a contradiction. 
Lemma 5.5. Let $G \leq S_{p^{k}}$, $p$ an odd prime and $k \geq 2$, be transitive with Sylow p-subgroup $P$ that is abelian. Let $P^{G}$ be the normal closure of $P$ in $G$. If $G$ admits a complete block system $\mathcal{B}$ such that $\operatorname{fix}_{P^{G}}(\mathcal{B})$ is semiregular and $P^{G} / \mathcal{B}$ is permutation isomorphic to $H \times K$ (with the canonical action), where $H$ is regular and abelian and $K$ is a direct product nonabelian simple groups, then $P^{G}$ is permutation isomorphic to $L \times K$ (with the canonical action) where $L$ is abelian.

Proof. As $\operatorname{fix}_{G}(\mathcal{B})$ is semiregular, $\operatorname{fix}_{G}(\mathcal{B})=\operatorname{fix}_{P}(\mathcal{B})$, and as $P^{G} / \mathcal{B}$ is permutation isomorphic to $H \times K, P^{G} / \mathcal{B}$ admits a complete block system $\mathcal{C}^{\prime}$ formed by the orbits of $H$. As the inverse image of a normal subgroup under a homomorphism is a normal subgroup, we have that if $L \leq P^{G}$ is maximal such that $L / \mathcal{B}=H$, then $L$ is a normal subgroup of $P^{G}$. As $\operatorname{fix}_{L}(\mathcal{B})=\operatorname{fix}_{G}(\mathcal{B})$ is a $p$-group and $L / \mathcal{B}=H$ is a $p$-group, we have that $L$ is a normal $p$-subgroup of $P^{G}$. As a Sylow $p$-subgroup of $G$ is abelian, we have that $L$ is abelian. Also, $P^{G}$ admits a complete block system $\mathcal{C}$ consisting of $p^{k-i}$ blocks of size $p^{i}$ induced by $\mathcal{C}^{\prime}$. That is, each block of $\mathcal{C}$ is the union of those blocks of $\mathcal{B}$ that are contained in a block of $\mathcal{C}^{\prime}$. Hence $\operatorname{fix}_{P^{G}}(\mathcal{C})=L$ and $P^{G} / \mathcal{C}=K$. By Lemma 5.1, we have that $L \leq Z\left(P^{G}\right)$. Note also that as $\mathcal{C}$ consists of $p^{k-i}$ blocks of size $p^{i}, K$ has degree $p^{k-i}$.

By Lemma 5.3, $\left(P^{G}\right)^{\prime} \cap \operatorname{fix}_{P^{G}}(\mathcal{C})=1$. Also, as $\operatorname{fix}_{P^{G}}(\mathcal{C})$ is a normal $p$-subgroup of $P^{G}$, fix $P_{P^{G}}(\mathcal{C})$ is contained in every Sylow $p$-subgroup of $P^{G}$. As $K$ is a direct product of nonabelian simple groups, we have that $\left(P^{G}\right)^{\prime} / \mathcal{C} \geq \operatorname{soc}(K)=K$ and is transitive. Let $P_{1}$ be a Sylow $p$-subgroup of $\left(P^{G}\right)^{\prime}$ and $P_{2}$ a Sylow $p$-subgroup of $P^{G}$ that contains $P_{1}$. Then $P_{2}$ is abelian, so $P_{1} \triangleleft P_{2}$ and $\operatorname{fix}_{P G}(\mathcal{C}) \triangleleft P_{2}$ as well as a normal $p$-subgroup is contained in every Sylow $p$-subgroup. Of course, $P_{1} \cap \operatorname{fix}_{P^{G}}(\mathcal{C})=1$ as $\left(P^{G}\right)^{\prime} \cap \operatorname{fix}_{P^{G}}(\mathcal{C})=1$, and, as $P_{1} / \mathcal{C}$ is transitive and $\left.\operatorname{fix}_{P^{G}}(\mathcal{C})\right|_{C}$ is transitive, $C \in \mathcal{C}$, we have that $\left\langle P_{1}\right.$, $\left.\operatorname{fix}_{P^{G}}(\mathcal{C})\right\rangle=P_{2}$, and so $P_{2} \cong P_{1} \times \operatorname{fix}_{P^{G}}(\mathcal{C})$. Thus $P_{1}$ has order $p^{k-i}$, and so no orbit of $P_{1}$ has order more than $p^{k-i}$.

Now, as $\left(P^{G}\right)^{\prime} \triangleleft P^{G}$, the orbits of $\left(P^{G}\right)^{\prime}$ form a complete block system $\mathcal{D}$ of $P^{G}$. Then $P_{1}$ is transitive on each block of $\mathcal{D}$ and as $P_{1}$ is abelian of order $p^{k-i}$, each block of $\mathcal{D}$ has order $p^{k-i}$. Thus $P_{1}$ is a Sylow $p$-subgroup of $\operatorname{fix}_{P^{G}}(\mathcal{D})$, and $\operatorname{fix}_{P^{G}}(\mathcal{D}) \cap \operatorname{fix}_{P^{G}}(\mathcal{C})=1$. By Lemma 5.4, $|C \cap D| \leq 1$ for every $C \in \mathcal{C}$ and $D \in \mathcal{D}$. As the number of possible intersections of blocks of $\mathcal{C}$ and $\mathcal{D}$ is $p^{k}$, we conclude that $|C \cap D|=1$ for every $C \in \mathcal{C}$ and $D \in \mathcal{D}$. By [6, Lemma 2.2], $P^{G}$ is permutation isomorphic to a subgroup of $S_{p^{k-i}} \times S_{p^{i}}$. Let $h \in P^{G}$. Then $h / \mathcal{C} \in K \cong \operatorname{fix}_{P G}(\mathcal{D})$. Thus $h \in \operatorname{fix}_{P G}(\mathcal{C}) \cdot K$, and as $K \cap \operatorname{fix}_{P G}(\mathcal{C})=$ 1 , we have that $h \in K \times \operatorname{fix}_{P^{G}}(\mathcal{C})$. The result then follows as $L=\operatorname{fix}_{P^{G}}(\mathcal{C})$.

Lemma 5.6. Let $G \leq S_{p^{k}}$, $k \geq 2$ such that a Sylow p-subgroup of $G$ is a regular abelian subgroup, and $G$ admits a nontrivial complete block system $\mathcal{B}$ such that $\operatorname{fix}_{G}(\mathcal{B})$ is semiregular and $G / \mathcal{B}$ is primitive. Then there exists $H \triangleleft G$ such that $H$ is permutation isomorphic to a subgroup of $S_{p^{i}} \times S_{p^{j}}$, where $p^{j}$ is the size of a block of $\mathcal{B}$, and $i+j=k$, or $P \triangleleft G$.

Proof. By [16, Theorem 1.1], we have that $\operatorname{soc}(G / \mathcal{B})$ is a direct product of nonabelian simple groups or is elementary abelian. If $\operatorname{soc}(G / \mathcal{B})$ is elementary abelian, then $G / \mathcal{B}$ contains a normal $p$-subgroup. As $\operatorname{fix}_{G}(\mathcal{B})$ is a $p$-group (as it is semiregular), we have that $G$ contains a transitive normal Sylow $p$-subgroup $Q$. As a Sylow $p$-subgroup of $G$ is a regular abelian group, $Q=P$ and the result follows. If $\operatorname{soc}(G / \mathcal{B})$ is a direct product of nonabelian simple groups, then first observe that $P^{G} / \mathcal{B}=(P / \mathcal{B})^{G / \mathcal{B}}$. Also, $\operatorname{soc}(G / \mathcal{B})$ contains a transitive $p$-subgroup which we may assume is $P / \mathcal{B}$. Thus every conjugate of $P / \mathcal{B}$ is contained in $\operatorname{soc}(G) / \mathcal{B}$. The result then follows by Lemma 5.5 with $H=P^{G}$. 


\section{The main results}

In this section, we have the final lemma that we will need to prove the main results, and then prove the main results.

Definition 6.1. Let $G$ be a group. The Frattini subgroup $\Phi(G)$ is the intersection of all proper maximal subgroups of $G$. Note that $\Phi(G)$ is equal to the set of all nongenerators of $G$, where a nongenerator of $G$ is an element $g$ such that if $X$ is a generating set of $G$, then $X-\{g\}$ is also a generating set of $G$.

Lemma 6.2. Let $G \leq S_{p^{\ell}}, p$ a prime and $\ell \geq 2$, be transitive such that a Sylow $p$ subgroup $P$ of $G$ is regular and abelian. Suppose that $G$ admits a complete block system $\mathcal{B}$ such that $\left.\operatorname{Stab}_{G}(B)\right|_{B}, B \in \mathcal{B}$, is primitive but $\left.\operatorname{fix}_{G}(\mathcal{B})\right|_{B}$ is imprimitive. If there exists $g \in \Phi(P)$ such that $g \in \operatorname{fix}_{G}(\mathcal{B})$ but there is no $h \in \operatorname{fix}_{P}(\mathcal{B})$ such that $\langle g\rangle\langle\langle h\rangle$, then $\left.\operatorname{fix}_{G}(\mathcal{B})\right|_{B} \leq \operatorname{AGL}(m, p)$, where $\mathcal{B}$ consists of blocks of size $p^{m}$.

Proof. As $\left.\operatorname{Stab}_{G}(B)\right|_{B}$ is primitive and contains a regular abelian subgroup, $\left.\operatorname{Stab}_{G}(B)\right|_{B}$ is given by [16, Theorem 1.1]. As fix $\left.\operatorname{fin}_{G}(\mathcal{B})\right|_{B}$ is imprimitive and $\left.\left.\operatorname{fix}_{G}(\mathcal{B})\right|_{B} \triangleleft \operatorname{Stab}_{G}(B)\right|_{B}$, $\left.\operatorname{Stab}_{G}(B)\right|_{B}$ cannot have simple socle. We conclude that $\left.\operatorname{Stab}_{G}(B)\right|_{B} \leq \operatorname{AGL}(m, p)$ (where $\mathcal{B}$ consists of blocks of size $p^{m}$ ), or is contained in $S_{r}<L$, where $L$ is a primitive almost simple group of degree $p^{m / r}$, and $r \geq 2$. In the former case, the result follows with no additional hypothesis as $\left.\operatorname{fix}_{G}(\mathcal{B})\right|_{B} \leq\left.\operatorname{Stab}_{G}(B)\right|_{B}$, so we need now only consider the case where $\left.\operatorname{Stab}_{G}(B)\right|_{B} \leq S_{r} \prec L$.

Let $g \in \Phi(P)$ such that $g \in \operatorname{fix}_{G}(\mathcal{B})$ but there is no $h \in \operatorname{fix}_{P}(\mathcal{B})$ such that $\langle g\rangle\langle\langle h\rangle$. As $P$ is a finite abelian group, we have that there exists $h \in P$ such that $\langle g\rangle\langle\langle h\rangle$. Thus $h / \mathcal{B} \neq 1$. Let $H=\left\langle P, \operatorname{fix}_{G}(\mathcal{B})\right\rangle$. Then $H / \mathcal{B}$ is a regular abelian group of degree $n / p^{m}$, and so the orbits of $\langle h\rangle / \mathcal{B}$ form a complete block system $\mathcal{C}^{\prime}$ of $H / \mathcal{B}$. Then $\mathcal{C}^{\prime}$ induces a complete block system $\mathcal{C}$ of $H$, where each block of $\mathcal{C}$ is the union of the blocks of $\mathcal{B}$ that are a block of $\mathcal{C}^{\prime}$. As $\left.\operatorname{Stab}_{G}(B)\right|_{B} \leq S_{r} \prec L$, we have that $T^{r} \leq\left.\operatorname{fix}_{G}(\mathcal{B})\right|_{B} \leq L^{r}$, where $T=\operatorname{soc}(L)$. Also note that as a Sylow $p$-subgroup of $G$ is regular, we must have that $\operatorname{fix}_{G}(\mathcal{B})$ acts faithfully on each $B \in \mathcal{B}$. Hence $\left.\operatorname{fix}_{G}(\mathcal{B}) \cong \operatorname{fix}_{G}(\mathcal{B})\right|_{B}$. Let $M$ be the smallest normal subgroup of $\operatorname{fix}_{G}(\mathcal{B})$ that contains $h$. Then $M=T^{t}$ for some $t \leq r$. As a Sylow $p$-subgroup of $T^{t}$ is isomorphic to $\mathbb{Z}_{p^{m / r}}^{t}$ and $\langle h\rangle$ is cyclic, we have that $t=1$. We conclude that $M$ has a normal complement $N$ in $T^{r}$, and that $N$ is normal in $H$. Let $\mathcal{F}$ be the complete block system of $H$ formed by the orbits of $N$. Then $A=\left.\left[\operatorname{fix}_{H}(\mathcal{C}) / \mathcal{F}\right]\right|_{C / \mathcal{F}}$, $C \in \mathcal{C}$, is imprimitive, has a cyclic Sylow $p$-subgroup, but does not have a normal Sylow $p$-subgroup, contradicting Theorem 1.2.

The following result generalizes the result of Jones [14] mentioned in the introduction.

Theorem 6.3. Let $G \leq S_{p^{k}}$ be transitive with an abelian Sylow p-subgroup P. Let $t$ be the rank of $P$. If $p>2^{t-1}$, then $P^{G}$ is permutation isomorphic to a direct product of cyclic groups and doubly-transitive nonabelian simple groups with the canonical action, with the number of factors in the direct product equal to the rank of $P$.

Proof. If $G$ is primitive, then by [16, Theorem 1.1], we have that $G \leq \operatorname{AGL}(k, p)$ or $G \leq S_{r} \prec N$, where $N$ is an almost simple group of degree $p^{k / r}$. If $G \leq \operatorname{AGL}(k, p)$, then $P \triangleleft G$ and the result follows. If $G \leq S_{r} \succ N$, then $\operatorname{soc}(G)=Q^{r}$ with the canonical action for some simple group $Q$ of degree $p^{k / r}$. As $Q^{r}$ is transitive, $p^{k}|| Q^{r} \mid$. As a Sylow $p$-subgroup of $G$ is $P$ and every Sylow $p$-subgroup of $G$ is conjugate, we have that 
$P \leq Q^{r}$, so that $P^{G}=Q^{r}$ and the result then follows as $Q$ is necessarily doubly-transitive by [11] as $Q$ contains an abelian Sylow $p$-subgroup. We henceforth assume without loss of generality that $G$ is imprimitive.

For the imprimitive case, we proceed by induction on $k$. If $k=2$, then the result can be deduced easily from [14, Proposition A and B] or [9, Theorem 4]. Let $k \geq 3$ and assume that the result holds for all $G$ satisfying the hypothesis of degree $p^{j}, j<k$.

Let $\mathcal{B}$ be a necessarily normal complete block system of $G$ such that there is no nontrivial complete block system $\mathcal{C}$ of $G$ such that $\mathcal{C} \prec \mathcal{B}$. Note that as usual, we must have that $\operatorname{fix}_{G}(\mathcal{B})$ acts faithfully on $B \in \mathcal{B}$ as a Sylow $p$-subgroup of $G$ is abelian. Also, $\left.\operatorname{Stab}_{G}(B)\right|_{B}$ is primitive by [5, Exercise 1.5.10]. We also let $\mathcal{B}$ consist of $p^{k-m}$ blocks of size $p^{m}$.

If $\left.\operatorname{fix}_{G}(\mathcal{B})\right|_{B}$ is primitive then by Lemma 4.3, we have that $G$ is permutation isomorphic to a subgroup of $S_{p^{k-m}} \times S_{p^{m}}$. Let $H \leq S_{p^{k-m}}$ and $K \leq S_{p^{m}}$ be minimal such that $G \leq H \times K$. Let $P_{1}$ be a Sylow $p$-subgroup of $H$ and $P_{2}$ a Sylow $p$-subgroup of $K$. As $H$ and $K$ are contained in appropriate projections of $G, P_{1}$ and $P_{2}$ are both regular abelian groups, and so $P=P_{1} \times P_{2}$. Thus the sum of the rank of $P_{1}$ and the rank of $P_{2}$ must be the same as the rank of $P$. By the induction hypothesis both $P_{1}^{H}$ and $P_{2}^{K}$ can be written as a direct product of cyclic groups and doubly-transitive nonabelian simple groups, with the number of factors in the direct product equal to rank of a Sylow $p$-subgroup of $H$ and $K$, respectively. By comments earlier in this paragraph, we conclude that the number of factors in the direct product of $P_{1}^{H}$ and $P_{2}^{K}$ is the rank of $P$. Finally, as both $H$ and $K$ are contained in appropriate projections of $G, P_{1}^{G}=P_{1}^{H}$ and $P_{2}^{G}=P_{2}^{K}$. Then $\left(P_{1} \times P_{2}\right)^{G}=P_{1}^{H} \times P_{2}^{K}$. The result then follows by the induction hypothesis.

If $\left.\operatorname{fix}_{G}(\mathcal{B})\right|_{B}$ is imprimitive and $\left.\operatorname{Stab}_{G}(\mathcal{B})\right|_{B} \leq \operatorname{AGL}(m, p)$, then by Lemma 5.1, we have that $\operatorname{fix}_{P}(\mathcal{B})$ is contained in the center of $P^{G}$ and $\left.\operatorname{fix}_{P^{G}}(\mathcal{B})\right|_{B} \leq\left.\operatorname{fix}_{P}(\mathcal{B})\right|_{B}$ for every $B \in \mathcal{B}$. As a Sylow $p$-subgroup of $\operatorname{fix}_{G}(\mathcal{B})$ is semiregular, we have that $\operatorname{fix}_{P^{G}}(\mathcal{B})=$ $\operatorname{fix}_{P}(\mathcal{B})$. By the induction hypothesis $P^{G} / \mathcal{B}$ can be written as a direct product of cyclic groups or doubly-transitive nonabelian simple groups. If $P^{G} / \mathcal{B}$ is abelian, then $P^{G}$ is abelian and $P \triangleleft G$, in which case the result follows. Otherwise, by Lemma 5.5, $P^{G}$ is permutation isomorphic to a direct product $L \times M$ with the canonical action, where $L$ is abelian, and $M$ is a direct product of nonabelian simple groups each of which is necessarily doubly-transitive by [11] as they all have abelian Sylow $p$-subgroups. It is then easy to see that the number of factors of $L \times M$ is the rank of $P$ as a Sylow $p$-subgroup of a doublytransitive nonabelian simple group of degree a prime-power is cyclic if it is abelian (this is implicit in [16, Theorem 1.1]), and the result follows by induction.

Finally, if $\left.\operatorname{fix}_{G}(\mathcal{B})\right|_{B}$ is imprimitive and $\left.\operatorname{Stab}_{G}(\mathcal{B})\right|_{B} \not \leq \operatorname{AGL}(m, p)$, then let $\mathcal{C}$ be a complete block system of $P^{G}$ such that $\mathcal{C} \preceq \mathcal{B}$ and there is no nontrivial complete block system $\mathcal{D}$ of $P^{G}$ such that $\mathcal{D} \prec \mathcal{C}$. Note that $\left.\operatorname{Stab}_{G}(B)\right|_{B} \leq S_{r} \prec K$, where $K$ is a doubly-transitive group with nonabelian socle $T$ of degree $p^{m / r}$. Then $T^{r} \triangleleft$ fix $_{G}(\mathcal{B})$, and $T^{r}$ is transitive on each block of $B \in \mathcal{B}$. Let $H=\left\langle T^{r}, P\right\rangle$, so that $H$ is transitive, and admits a complete block system consisting of blocks of size $p^{m / r}$ formed by the orbits of a factor of $T^{r}$ (note that this is true as $\operatorname{Stab}_{H}(B)=\operatorname{fix}_{H}(\mathcal{B})=T^{r}$ for every $B \in \mathcal{B}$ ). By arguments above, $H$ is a direct product of cyclic groups and doubly-transitive nonabelian simple groups, with the number of factors equal to the rank of $P$. As every doubly-transitive nonabelian simple group factor of $H$ is contained in $\operatorname{fix}_{H}(\mathcal{B})$, we conclude that $r<t$. By Lemma 3.6, we conclude that $\mathcal{C} \prec \mathcal{B}$.

Now, $P^{G}$ is the subgroup of $G$ generated by all of the Sylow $p$-subgroups of $G$, and 
$P^{P^{G}}$ is the subgroup generated by all of the Sylow $p$-subgroups of $P^{G}$. As $P^{G}$ contains every Sylow $p$-subgroup of $G, P^{G}=P^{P^{G}}$. We conclude by choice of $\mathcal{C}$ and Lemma 3.6 that $\left.\operatorname{fix}_{P G}(\mathcal{C})\right|_{C}$ is either primitive or contained in $\operatorname{AGL}(n, p)$ for some $n$. In either case, the result follows by arguments above and the fact that $P^{P^{G}}=P^{G}$.

The following result is a special case of the previous result.

Corollary 6.4. Let $G \leq S_{p^{k}}$, $p$ an odd prime and $k \geq 2$, be transitive with Sylow $p$ subgroup $P$ that is abelian of rank two. Let $P^{G}$ be the normal closure of $P$ in $G$. Then $P^{G} \triangleleft G$ is permutation isomorphic to $L \times M$ with the canonical action, where $L \leq S_{p^{\ell}}$, $M \leq S_{p^{m}}$, are either regular cyclic groups or doubly-transitive simple groups.

If $P$ is of rank two, we can obtain a complete description of the transitive groups of odd prime-power degree that have $P$ as a Sylow $p$-subgroup.

Corollary 6.5. Let $G \leq S_{p^{k}}$, $p$ an odd prime and $k \geq 2$, be transitive with Sylow $p$ subgroup $P$ that is abelian of rank two. Then one of the following is true:

1. $G \leq N_{S_{p^{k}}}(P)$,

2. $k$ is even, $G$ is primitive and $G \leq S_{2} 2 T$, where $T$ is a doubly-transitive group with nonabelian socle of degree $p^{k / 2}$ with cyclic Sylow $p$-subgroup, or

3. there exists $H \leq S_{p^{\ell}}, K \leq S_{p^{m}}$, $\ell+m=k$, each of whose Sylow p-subgroups is cyclic, and $H \times K \leq G \leq N_{S_{p \ell}}(H) \times N_{S_{p} m}(K)$, where $H$ is doubly-transitive and simple and $K$ is either a doubly-transitive and simple or is cyclic.

Proof. By Theorem 6.4, either the result follows or $P^{G}$ is permutation isomorphic to $H \times K$ with the canonical action, where $H$ and $K$ are transitive permutation groups of degree $p^{\ell}$ and $p^{m}$, respectively, $\ell+m=k$, and at least one of $H$ or $K$, say $H$, is a doubly-transitive simple group, and if $K$ is not a doubly-transitive simple group, then it is cyclic. If $G$ is primitive, then $\operatorname{soc}(G)$ must be a direct product of isomorphic nonabelian simple groups $L$, and $G \leq S_{r} \imath T, r \geq 2$, for some nonabelian almost simple group $T$ by [16, Theorem 1.1]. As a Sylow $p$-subgroup of $G$ is abelian of $\operatorname{rank}$ two, $\operatorname{soc}(G)$ must be a direct product of two isomorphic nonabelian simple groups, and so $r=2$. Thus the result follows if $G$ is primitive.

If $G$ is imprimitive, then either both $H$ and $K$ are doubly-transitive simple groups, or only $H$ is. In the latter case, $P^{G}=H \times \mathbb{Z}_{p^{m}}$, so that $Z\left(P^{G}\right)=1_{H} \times \mathbb{Z}_{p^{m}}$ and $\left(P^{G}\right)^{\prime}=H \times 1_{\mathbb{Z}_{p^{m}}}$. As both $Z\left(P^{G}\right)$ and $\left(P^{G}\right)^{\prime}$ are characteristic, we have that $Z\left(P^{G}\right)$ and $\left(P^{G}\right)^{\prime}$ are normal in $G$. Thus $G$ admits complete block systems $\mathcal{B}$ and $\mathcal{C}$ of $p^{m}$ blocks of size $p^{\ell}$ and $p^{\ell}$ blocks of size $p^{m}$, respectively, formed by the orbits of $\left(P^{G}\right)^{\prime}$ and $Z\left(P^{G}\right)$, respectively. Clearly the intersection of a block of $\mathcal{B}$ and a block of $\mathcal{C}$ is a singleton, and so by [6, Lemma 2.2] we have that $G$ is permutation isomorphic to a subgroup of $S_{p^{\ell}} \times S_{p^{m}}$. It is then not difficult to see that $G \leq N_{S_{p} \ell}(H) \times N_{S_{p} m}(K)$ and the result follows. We thus assume that both $H$ and $K$ are doubly-transitive simple groups.

Clearly $H \times K$ admits $\mathcal{B}$ and $\mathcal{C}$ as complete block systems, where $\mathcal{B}$ and $\mathcal{C}$ are formed by the orbits of $H \times 1_{K}$ and $1_{H} \times K$, respectively. Thus $\mathcal{B}$ consists of $p^{m}$ blocks of size $p^{\ell}$ and $\mathcal{C}$ consists of $p^{\ell}$ blocks of size $p^{m}$, and the intersection of a block of $\mathcal{B}$ with a block of $\mathcal{C}$ is a singleton. Let $\mathcal{D}$ be a nontrivial complete block system of $H \times K$. As $H \times K$ contains a regular abelian group, $\operatorname{fix}_{H \times K}(\mathcal{D}) \neq 1$, so that $\operatorname{fix}_{H \times K}(\mathcal{D})=H \times 1_{K}$ or $K \times 1_{H}$, as these are the only proper nontrivial normal subgroups of $H \times K$. Thus $\mathcal{D}=\mathcal{B}$ or $\mathcal{C}$. If 
$g \in G$, then $g(\mathcal{B})$ and $g(\mathcal{C})$ are complete block systems of $g(H \times K) g^{-1}=H \times K$ so that $g(\mathcal{B})=\mathcal{B}$ and $g(\mathcal{C})=\mathcal{C}$ or $g(\mathcal{B})=\mathcal{C}$ and $g(\mathcal{C})=\mathcal{B}$. Note that if $g(\mathcal{B})=\mathcal{C}$ and $g(\mathcal{C})=\mathcal{B}$, then $G$ has no nontrivial complete block systems, and so $G$ is primitive, a contradiction. Otherwise, $\mathcal{B}$ and $\mathcal{C}$ are complete block systems of $G$ and so by [6, Lemma 2.2] we have that $G$ is permutation isomorphic to a subgroup of $S_{p^{\ell}} \times S_{p^{m}}$ as above. Again, we conclude that $G \leq N_{S_{p^{\ell}}}(H) \times N_{S_{p^{m}}}(K)$ and the result follows.

Remark 6.6. Corollary 6.5 (2) is a special case of [16, Theorem 1.1].

Remark 6.7. If (2) occurs in the previous result, then as a Sylow $p$-subgroup of $G$ is abelian of rank two, a Sylow $p$-subgroup of $T$ must by cyclic. Similarly, if (3) occurs and either $H$ or $K$ is doubly-transitive with nonabelian socle, then a Sylow $p$-subgroup of $H$ or $K$ is also cyclic. As every minimal transitive subgroup of a group of prime-power degree is a $p$-group [19, Theorem 3.4], any Sylow $p$-subgroup of $T$ and $H$ or $K$ as above are transitive. Thus $T$ and $H$ or $K$ are given by Theorem 1.2.

We would like to make the following conjecture.

Conjecture 6.8. Let $G \leq S_{p^{k}}$ be transitive with an abelian Sylow p-subgroup P. Then $P^{G}$ is permutation isomorphic to a direct product of cyclic groups and doubly-transitive nonabelian simple groups, with the number of factors in the direct product equal to the rank of $P$.

\section{$7 \quad$ 2-closed groups}

One of the main motivations for this work is to help in determining the full automorphism groups of Cayley digraphs of abelian $p$-groups (Cayley digraphs are defined immediately after this paragraph), which necessarily contain a transitive abelian subgroup. When determining such full automorphism groups, one of the more difficult cases seems to be when the Sylow $p$-subgroup of the full automorphism group is small - and for a Cayley digraph of an abelian $p$-group, the smallest possible Sylow $p$-subgroup is a transitive abelian group. We now determine the full automorphism groups of Cayley digraphs of abelian groups of rank two, and do so in the more general context of a 2-closed group. At this time, in order to determine the full automorphism groups of Cayley digraphs of abelian groups whose automorphism group contains a regular abelilan subgroup of rank more than two, more explicit information will be needed about the such digraphs whose automorphism group is primitive. This problem will be studied in [8].

Definition 7.1. Let $G$ be a group and $S \subset G$. Define a Cayley digraph of $G$, denoted $\Gamma(G, S)$ to be the digraph with $V(\Gamma(G, S))=G$ and $E(\Gamma(G, S))=\{(g, g s): g \in$ $G, s \in S\}$. We remark that if $\Gamma(G, S)$ is a Cayley digraph of $G$, then $G_{L} \leq \operatorname{Aut}(\Gamma)$. If $S=S^{-1}$, then $\Gamma(G, S)$ is a Cayley graph of $G$. A vertex-transitive digraph is a digraph whose automorphism group acts transitively on the vertices of the graph. Note that $G_{L}$ is transitive on $G$, so every Cayley digraph is vertex-transitive.

We now define the 2-closure of a permutation group $G$.

Definition 7.2. Let $\Omega$ be a set and $G \leq S_{\Omega}$. Let $G$ act on $\Omega \times \Omega$ by $g\left(\omega_{1}, \omega_{2}\right)=$ $\left(g\left(\omega_{1}\right), g\left(\omega_{2}\right)\right)$ for every $g \in G$ and $\omega_{1}, \omega_{2} \in \Omega$. We define the 2 -closure of $G$, denoted $G^{(2)}$, to be the largest subgroup of $S_{\Omega}$ whose orbits on $\Omega \times \Omega$ are the same as $G$ 's. Let 
$\mathcal{O}_{1}, \ldots, \mathcal{O}_{r}$ be the orbits of $G$ acting on $\Omega \times \Omega$. Define digraphs $\Gamma_{1}, \ldots, \Gamma_{r}$ by $V\left(\Gamma_{i}\right)=\Omega$ and $E\left(\Gamma_{i}\right)=\mathcal{O}_{i}$. Each $\Gamma_{i}, 1 \leq i \leq r$, is an orbital digraph of $G$, and it is straightforward to show that $G^{(2)}=\cap_{i=1}^{r} \operatorname{Aut}\left(\Gamma_{i}\right)$. Clearly the automorphism group of a vertex-transitive graph or digraph is 2-closed.

Corollary 7.3. Let $G \leq S_{p^{k}}$ be transitive and 2-closed with Sylow p-subgroup $P$ that is abelian of rank two. Then one of the following is true:

1. G has a normal Sylow p-subgroup,

2. $G$ is primitive, $k=2$, and $G$ is permutation isomorphic to $S_{2} \prec S_{p}$,

3. $k=2$, and $G$ is permutation isomorphic to $S_{p} \times S_{p}$, or

4. $G$ is permutation isomorphic to $S_{p} \times A$, where $A \leq N\left(p^{k-1}\right)$ has order dividing $(p-1) p^{k-1}$.

Proof. Suppose $G$ does not have a normal Sylow $p$-subgroup. By Corollary 6.5, either $k$ is even, $G$ is primitive, and $G \leq S_{2} \succ T$, where $T$ is a nonabelian doubly-transitive group of degree $p^{k / 2}$ with cyclic Sylow $p$-subgroup, or there exists $H \leq S_{p^{\ell}}, K \leq S_{p^{m}}, \ell+m=k$, each of whose Sylow $p$-subgroups is cyclic, and $H \times K \leq G \leq N_{S_{p^{\ell}}}(H) \times N_{S_{p} m}(K)$, and $H$ and $K$ are either doubly-transitive simple groups or are cyclic. By [15] (this result also appears in [4, Theorem 5.1]), we have that if $H$ or $K$ is doubly-transitive, then, as the 2-closure of a doubly-transitive group is a symmetric group, $H=S_{p^{\ell}}$ or $K=S_{p^{m}}$. By the same result, we must also have that $T$ is a symmetric group. We conclude that if $H, K$, or $T$ is doubly-transitive, then as a Sylow $p$-subgroup of $G$ is abelian, we must have that $\ell=1$ or $m=1$. In the primitive case, we then have that $G=S_{2} \imath S_{p}$ and the result follows. If both $H$ and $K$ are doubly-transitive, then clearly $G=S_{p} \times S_{p}$, and if both $H$ and $K$ are cyclic, then $G$ has a normal Sylow $p$-subgroup. If exactly one of $H$ and $K$ is doubly-transitive, say $H$, then $S_{p} \times \mathbb{Z}_{p^{k-1}} \leq G \leq N_{S_{p}}\left(S_{p}\right) \times N_{S_{p^{k-1}}}\left(\mathbb{Z}_{p^{k-1}}\right)=S_{p} \times N_{S_{p^{k-1}}}\left(\mathbb{Z}_{p^{k-1}}\right)$, so that $G=S_{p} \times A$, where $A \leq N_{S_{p^{k-1}}}\left(\mathbb{Z}_{p^{k-1}}\right)=N\left(p^{k-1}\right)$. Finally, $|A| \mid(p-1) p^{k-1}$ as $\left|N\left(p^{k-1}\right)\right|=(p-1) p^{k-2} \cdot p^{k-1}$ and a Sylow $p$-subgroup of $A$ must have order $p^{k-1}$ as a Sylow $p$-subgroup of $G$ has order $p^{k}$.

\section{References}

[1] B. Alspach and T. D. Parsons, Isomorphism of circulant graphs and digraphs, Discrete Math. 25 (1979), no. 2, 97-108.

[2] W. Burnside, On some properties of groups of odd order, J. London Math. Soc. 33 (1901), $162-185$.

[3] P. J. Cameron, Finite Permutation groups and finite simple groups, Bull. London Math. Soc. 13 (1981), 1-22.

[4] P. J. Cameron, M. Giudici, W. M. Kantor, G. A. Jones, M. H. Klin, D. Marušič and L. A. Nowitz, Transitive permutation groups without semiregular subgroups, J. London Math. Soc. 66 (2002), 325-333.

[5] J. D. Dixon and B. Mortimer, Permutation Groups, Springer-Verlag New York, Berlin, Heidelberg, Graduate Texts in Mathematics, 163, 1996.

[6] E. Dobson, Automorphism groups of metacirculant graphs of order a product of two distinct primes, Combin. Prob. Comput. 15 (2006), 105-130. 
[7] E. Dobson, On groups of odd prime-power degree that contain a full cycle, Discrete Mathematics 299 (2005), 65-78.

[8] E. Dobson and I. Kovács, Automorphism groups of Cayley digraphs of $\mathbb{Z}_{p}^{3}$, in preparation.

[9] E. Dobson and D. Witte, Transitive permutation groups of prime-squared degree, J. Algebraic Combin. 16 (2002), 43-69.

[10] C. D. Godsil, On Cayley graph isomorphisms, Ars Combin. 15 (1983), 231-246.

[11] R. M. Guralnick, Subgroups of prime power index in a simple group, J. of Algebra 81 (1983), 304-311.

[12] T. Hungerford, Algebra, Holt, Rinehart and Winston, 1974.

[13] B. Huppert, Endliche Gruppen I, Springer, Berlin, 1967.

[14] G. A. Jones, Abelian subgroups of simply primitive groups of degree $p^{3}$, where $p$ is prime, Quart. J. Math. Oxford 30 (1979), no. 2, 53-76.

[15] L. A. Kalužnin and M. H. Klin, On some numerical invariants of permutation groups, Latviǔsk. Mat. Ežegodnik 18 (1976), 81-99.

[16] C. H. Li, The finite primitive permutation groups containing an abelian regular subgroup, Proc. London Math. Soc. 87 (2003), no. 3, 725-747.

[17] M. W. Liebeck, C. E. Praeger and J. Saxl, On the O'Nan-Scott Theorem for finite primitive permutation groups, J. Austral. Math. Soc. Ser. A 44 (1988), 389-396.

[18] G. Sabidussi, The composition of graphs, Duke Math J. 26 (1959), 693-696.

[19] H. Wielandt, Finite Permutation Groups, Academic Press, New York, 1964.

[20] H. Wielandt, Permutation groups through invariant relations and invariant functions, lectures given at The Ohio State University, Columbus, Ohio, 1969. 